\title{
Ações educativas desenvolvidas por enfermeiros para a segurança dos pacientes no
}

\section{perioperatório}

\author{
Educational actions developed by nurses for patient safety in the perioperative period \\ Acciones educativas desarrolladas por enfermeiras para la seguridade del paciente em el período \\ perioperatorio
}

Recebido: 12/04/2021 | Revisado: 17/04/2021 | Aceito: 23/04/2021 | Publicado: 23/07/2021

Vivian Lemes Lobo Bittencourt

ORCID: https://orcid.org/0000-0003-1488-0611 Universidade Regional do Noroeste do Estado do Rio Grande do Sul, Brasil E-mail: vivillobo@hotmail.com

Maria Simone Vione Schwengber ORCID: https://orcid.org/0000-0002-3583-1408 Universidade Regional do Noroeste do Estado do Rio Grande do Sul, Brasil E-mail: simone@unijui.edu.br

Eniva Miladi Fernandes Stumm

ORCID: https://orcid.org/0000-0001-6169-0453 Universidade Regional do Noroeste do Estado do Rio Grande do Sul, Brasil E-mail: eniva@unijui.edu.b

\begin{abstract}
Resumo
Esse artigo objetiva mapear estudos sobre ações educativas acerca da segurança do paciente no perioperatório, no decorrer da hospitalização. Trata-se de uma revisão narrativa a partir do mapeamento das produções de enfermeiros, em teses e dissertações brasileiras. Foram desenvolvidas cinco buscas no dia 15 de dezembro de 2020 no Catálogo de Teses e Dissertações da Coordenação de Aperfeiçoamento de Pessoal de Nível Superior, com combinações variadas dos descritores e/ou palavras-chave: paciente, cirúrgico, educação de pacientes como assunto, educação em saúde, segurança do paciente, enfermagem, enfermagem perioperatória, período pré-operatório, período pós-operatório, hospital. O operador booleano "AND" foi utilizado para interligar os termos nas buscas. Nove produções foram selecionadas e analisadas. Identificou-se várias percepções positivas de pacientes referentes a ações educativas desenvolvidas, valorização de informações sobre a hospitalização, cirurgias e qualidade dos variados temas abordados para a segurança dos pacientes. A pesquisa possibilitou conhecer estudos que tematizam ações educativas acerca da segurança dos pacientes no perioperatório desenvolvidas na hospitalização, porém não deixam claro se os pacientes relacionam as ações educativas em saúde com a segurança. A análise dos estudos que integraram essa revisão demonstra a lacuna de investigações que contemplem ações educativas por enfermeiros direcionadas à segurança do paciente cirúrgico.
\end{abstract}

Palavras-chave: Pacientes; Educação de pacientes como assunto; Educação em saúde; Enfermagem.

\begin{abstract}
This article aims to map studies on educational actions about patient safety in the perioperative period, during hospitalization. It is a narrative review based on the mapping of nurses productions, in Brasilian theses and dissertations. Five searches were carried out on december 15, 2020 in the Catalog of Theses and Dissertations of the Coordination for the Improvement of Higher Education Personnel, with varying combinations of descriptors and/or keywords: patient, surgical, patient education as a subject, education in health, patient safety, nursing, perioperative nursing, preoperative period, postoperative period, hospital. The boolean operator "AND" was used to interconnect search terms. Nine productions were selected and analyzed. Several positive perceptions of patients were identified regarding the educational sctions developed, valuing information on hospitalization, surgeries and the quality of various topics addressed for patient safety. The research made it possible to learn about studies that focus on educational actions about patient safety in the perioperative period developed during hospitalization, but they do not make in clear whether patients relate educational actions in health to safety. The analysis of the studies that integrated this review demonstrates the lack of investigations that include educational actions by nurses aimed at the safety of surgical patients.
\end{abstract}

Keywords: Patients; Patients education as topic; Health education; Nursing.

\section{Resumen}

Este artículo tiene como objetivo mapear estudios sobre acciones educativas sobre seguridad del paciente en el período perioperatorio, durante la hospitalización. Es una revisión narrativa basada em el mapeo de las producciones 
de enfermeras, em tesis y disertaciones brasileñas. El 15 de diciembre de 2020 se realizaron cinco búsquedas em el Catálogo de Tesis y Disertaciones de la Coordinación para el Perfeccionamiento del Personal de Educación Superior, con diversas combinaciones de descriptores y/o palabras clave: paciente, quirúrgico, educación del paciente como asignatura, educación em salud, seguridad del paciente, enfermería, enfermería perioperatoria, período preoperatorio, período postoperatorio, hospital. El operador booleano "Y" se utilizó para interconectar términos de búsqueda. Se seleccionaron y analizaron nueve producciones. Se identificaron varias percepciones positivas de los pacientes respecto a las acciones educativas desarrolladas, valorando la información sobre hospitalización, cirugías y la calidad de los diversos temas abordados para la seguridad del paciente. La investigación permitió conocer estudios que se centran en acciones educativas sobre seguridad del paciente en el período perioperatorio desarrollados durante la hospitalización, pero no aclaran si los pacientes relacionan las acciones educativas em salud con la seguridad. El análisis de los estudios que integraron esta revisión demuestra la falta de investigaciones que incluyan acciones educativas por parte de enfermeras dirigidas a la seguridad de los pacientes quirúrgicos.

Palabras clave: Pacientes; Educación de los pacientes como temas; Educación en salud; Enfermería.

\section{Introdução}

O paciente, ao ser hospitalizado para ser submetido a uma cirurgia, se depara com um ambiente não habitual, confronta medos, inseguranças, crendices, tem relação direta com profissionais de saúde com os quais não possui contato prévio, com exceção, talvez, do médico-cirurgião e anestesiologista, quando se trata de procedimentos cirúrgicos eletivos. A ambientação desse paciente pode ser favorecida com comunicação assertiva por meio de ações educativas (Böck et al., 2019). As referidas ações educativas podem ser entendidas como uma construção conjunta entre paciente e profissional de saúde, nesse texto pontualmente os enfermeiros, pautados na construção dos saberes para constituir, em relações horizontais, um sujeito ativo no processo de elaboração de conhecimento (Vasconcelos, 2019).

A participação do paciente em prol de sua segurança na assistência à saúde é um tema relevante para a prevenção e redução de eventos adversos, promoção de melhorias que impactem positivamente em sua experiência. A participação ativa do paciente foi estimulada, sobretudo mundialmente, a partir de 2008, com ações promovidas pela Aliança Mundial pela Segurança do Paciente frente à necessidade de ampliar a abrangência de ações para a redução da ocorrência de danos desnecessários associados à assistência à saúde (WHO, 2008). A publicação "Forward Programme 2008-2009” (2008) buscou, entre outros eixos, promover inovação e sustentar o compromisso ao apresentar o componente Pacientes pela Segurança dos Pacientes, que objetiva garantir que as vozes de pacientes sejam a base para um movimento nos ambientes de cuidado a saúde (WHO, 2008).

A experiência e o conhecimento de pacientes que vivenciaram incidentes relacionados à segurança podem ser vistos como fundamentais por enfermeiros, para a melhoria dos cuidados de saúde. Além disso, essas ocorrências podem potencializar o planejamento de ações educativas para que outros eventos adversos sejam prevenidos (WHO, 2008; BRASIL, 2014). Aliado ao conhecimento das ocorrências institucionais anteriores, a participação do paciente cirúrgico em ações educativas amplia seu conhecimento para o cuidado seguro pela construção de uma parceria segura e que motiva ambos os envolvidos, para a promoção da segurança no perioperatório.

Os enfermeiros idealizam que as ações educativas no processo de enfermagem de pacientes cirúrgicos favorecem, de modo particular, a comunicação entre paciente e enfermeiro, auxiliam na prevenção de situações indesejadas e que possam comprometer a integridade do sujeito. Assim, as ações educativas têm como base o diálogo, a troca de saberes, intercâmbio entre saber científico e popular, em que ambos os atores têm muito a ensinar e aprender (VASCONCELOS, 2019). Considerase significativo retomar que ao utilizar-se a denominação popular não se refere ao público ao qual se destina a ação educativa, mas sim aos desígnios éticos e às metodologias que a norteiam pela valorização dos saberes, construção compartilhada do conhecimento para o fortalecimento democrático (Vasconcelos; Cruz; Prado, 2016).

As ações educativas fazem sentido na hospitalização pelos potenciais riscos envolvidos e pela possibilidade de estimular o paciente como vigilante da segurança assistencial. Com essa revisão, busca-se demonstrar a lacuna referente à 
produção científica que envolve ações educativas na hospitalização de pacientes no perioperatório sobre a segurança. Em face disso, esse estudo objetiva mapear estudos sobre ações educativas acerca da segurança do paciente no perioperatório, no decorrer da hospitalização.

\section{Metodologia}

Trata-se de uma revisão narrativa da literatura, a partir do mapeamento das produções de enfermeiros, em teses e dissertações brasileiras. Essa modalidade de revisão possui caráter amplo e propõe descrever, sob o ponto de vista teórico ou contextual, determinado tema, com base no conjunto de produçães existentes, o que favorece a identificação de lacunas no conhecimento e a proposição de outros estudos (Brum et al., 2016).

A elaboração da questão de revisão foi inspirada na estrutura População-Interesse-Contexto (PICo), que descreve componentes relacionados ao problema identificado, elemento fundamental para a busca de evidências. A pesquisa teve como questão: quais são as ações educativas dos enfermeiros (I) desenvolvidas na hospitalização (Co) para a segurança dos pacientes no perioperatório $(\mathrm{P})$ ? Foi estabelecido como critério de inclusão pesquisas que contemplem a participação ativa de pacientes adultos hospitalizados no perioperatório, como sujeitos. Foi adotado como critério de exclusão não estar disponível eletronicamente. Os estudos encontrados em duplicidade foram contabilizados apenas uma vez.

As buscas ocorreram no dia 15 de dezembro de 2020. A seleção das pesquisas foi feita mediante busca no portal de Catálogo de Teses e Dissertações da Coordenação de Aperfeiçoamento de Pessoal de Nível Superior (Capes, disponível em http://bancodeteses.capes.gov.br), por meio da Comunidade Acadêmica Federada (CAFe).

Para a definição de descritores e/ou palavras-chave que iriam compor essa pesquisa de revisão narrativa da literatura foi desenvolvida uma busca breve no Portal Regional da Biblioteca Virtual de Saúde (BVS), com a utilização das palavraschave "(ações educativas) AND enfermeiro AND paciente", para a identificação das designações que melhor se enquadrariam em buscas amplas que possibilitassem a identificação do tema. A partir da leitura desses textos, foram organizados os descritores e/ou palavras-chave: paciente, cirúrgico, educação de pacientes como assunto, educação em saúde, segurança do paciente, enfermagem, enfermagem perioperatória, período pré-operatório, período pós-operatório, hospital. O operador booleano "AND" foi utilizado para interligar os termos nas buscas. Foram desenvolvidas cinco buscas com estruturações diferentes entre descritores e/ou palavras-chave para a obtenção do maior número de teses e dissertações sobre o tema (Tabela 1). 
Tabela 1 - Descritores utilizados na seleção e resultados obtidos de teses e dissertações. Ijuí, RS, Brasil, 2021.

\begin{tabular}{|c|c|c|c|c|c|}
\hline Descritores & $\begin{array}{c}\text { Total de } \\
\text { teses e } \\
\text { dissertações } \\
\text { encontradas }\end{array}$ & $\begin{array}{l}\text { Total após a } \\
\text { exclusão de teses e } \\
\text { dissertações não } \\
\text { disponíveis } \\
\text { eletronicamente }\end{array}$ & $\begin{array}{l}\text { Total após a } \\
\text { retirada de } \\
\text { duplicados }\end{array}$ & $\begin{array}{l}\text { Selecionados } \\
\text { para leitura na } \\
\text { íntegra }\end{array}$ & $\begin{array}{l}\text { Selecionados } \\
\text { para compor a } \\
\text { revisão }\end{array}$ \\
\hline $\begin{array}{l}\text { "Educação em saúde" AND } \\
\text { "segurança do paciente" AND } \\
\text { enfermagem AND hospital } \\
\text { AND paciente AND cirúrgico }\end{array}$ & 61 & 51 & 50 & 8 & 1 \\
\hline $\begin{array}{l}\text { "educação de pacientes como } \\
\text { assunto" }\end{array}$ & 9 & 9 & 9 & 2 & 1 \\
\hline $\begin{array}{l}\text { "Educação em saúde" AND } \\
\text { "segurança do paciente" AND } \\
\text { "enfermagem perioperatória" } \\
\text { AND hospital AND paciente } \\
\text { AND cirúrgico }\end{array}$ & 17 & 14 & 13 & 0 & 0 \\
\hline $\begin{array}{l}\text { "Educação em saúde" AND } \\
\text { enfermagem AND "Pré- } \\
\text { operatório" AND Paciente } \\
\text { AND "Educação de pacientes } \\
\text { como assunto" }\end{array}$ & 104 & 83 & 82 & 13 & 4 \\
\hline $\begin{array}{l}\text { "Educação em saúde" AND } \\
\text { enfermagem AND "Pós- } \\
\text { operatório" AND Paciente } \\
\text { AND "Educação de pacientes } \\
\text { como assunto" }\end{array}$ & 130 & 96 & 90 & 5 & 3 \\
\hline
\end{tabular}

Legenda: tese (T), dissertação (D). Fonte: Bittencourt; Schwengber \& Stumm (2021).

Foram encontradas, no total das cinco buscas, 321 pesquisas e, após a aplicação dos critérios de inclusão e exclusão, foram selecionadas nove pesquisas, que respondiam a questão elencada e que assim, constituíram o corpus de análise. A produção de dados ocorreu mediante aplicação de formulário específico construído pelas autoras com as seguintes variáveis: título, autores, ano de publicação, modalidade, região geográfica, instituição de ensino, área de concentração, abordagem metodológica, tipo de pesquisa, objetivo da pesquisa, período cirúrgico em que a ação foi desenvolvida, ação educativa desenvolvida, percepções de pacientes sobre a ação educativa, relação com a segurança, resultados e palavraschave/descritores.

Os dados foram analisados de forma qualitativa e descritiva, digitados em formulário próprio mediante as informações obtidas (BRUM et al., 2016). Por se tratar de uma revisão narrativa com a utilização de dados secundários, de domínio público, essa pesquisa não foi submetida ao Comitê de Ética em Pesquisa, entretanto, os princípios éticos em pesquisa foram garantidos.

\section{Resultados e Discussão}

Foram considerados nove trabalhos relacionados às ações educativas dos enfermeiros que são desenvolvidas na hospitalização para a segurança do paciente cirúrgico, sendo cinco dissertações $(55,6 \%)$ e quatro teses $(44,4 \%)($ Tabela 2$)$. 
Tabela 2 - Teses e dissertações de enfermagem brasileira sobre ações educativas acerca da segurança dos pacientes no perioperatório desenvolvidas na hospitalização. Ijuí, RS, Brasil, 2021.

\begin{tabular}{|c|c|c|c|c|}
\hline $\begin{array}{c}\text { Descritores/ } \\
\text { palavras-chave }\end{array}$ & Tipo & Ano & Título/Autor(a) & IES \\
\hline $\begin{array}{l}\text { "Educação em saúde" } \\
\text { AND "segurança do } \\
\text { paciente" AND } \\
\text { enfermagem AND } \\
\text { hospital AND pacientes } \\
\text { AND cirúrgico }\end{array}$ & $\mathrm{T}$ & 2017 & $\begin{array}{l}\text { Efetividade da cartilha “cirurgia bariátrica: } \\
\text { cuidados para uma vida saudável” no preparo pré- } \\
\text { operatório: ensaio clínico randomizado (BARROS, } \\
\text { 2017) }\end{array}$ & $\begin{array}{c}\text { Universidade } \\
\text { Federal do Ceará }\end{array}$ \\
\hline $\begin{array}{l}\text { "Educação de pacientes } \\
\text { como assunto" }\end{array}$ & $\mathrm{T}$ & 2016 & $\begin{array}{l}\text { Tecnologias Educacionais na Assistência } \\
\text { Perioperatória em Cirurgia Ortopédica no Contexto } \\
\text { da Consulta de Enfermagem (PINTO, 2016) }\end{array}$ & $\begin{array}{l}\text { Universidade } \\
\text { Federal do Rio de } \\
\text { Janeiro }\end{array}$ \\
\hline \multirow{4}{*}{$\begin{array}{l}\text { "Educação em saúde" } \\
\text { AND enfermagem } \\
\text { AND "Pré-operatório" } \\
\text { AND Paciente AND } \\
\text { "Educação de pacientes } \\
\text { como assunto" }\end{array}$} & $\mathrm{D}$ & 2013 & $\begin{array}{c}\text { Influência do processo educativo de Enfermagem } \\
\text { no pré e pós-operatório de paciente de cirurgia } \\
\text { cardíaca } \\
\text { (ROSSETO, 2013) }\end{array}$ & $\begin{array}{l}\text { Universidade } \\
\text { Federal do Espírito } \\
\quad \text { Santo }\end{array}$ \\
\hline & $\mathrm{D}$ & 2014 & $\begin{array}{l}\text { Cartilha com orientações de enfermagem para a alta } \\
\text { hospitalar: contribuição à educação em saúde do } \\
\text { paciente transplantado renal' (BORSATO, 2014) }\end{array}$ & $\begin{array}{c}\text { Universidade } \\
\text { Federal Fluminense }\end{array}$ \\
\hline & $\mathrm{T}$ & 2013 & $\begin{array}{l}\text { Efetividade de um programa de ensino para o } \\
\text { cuidado domiciliar de pacientes submetidos à } \\
\text { prostatectomia radical: ensaio clínico randomizado' } \\
\text { (MATA, 2013) }\end{array}$ & $\begin{array}{l}\text { Universidade de } \\
\quad \text { São Paulo }\end{array}$ \\
\hline & $\mathrm{T}$ & 2016 & $\begin{array}{l}\text { Avaliação do uso de uma tecnologia educativa na } \\
\text { educação perioperatória de cirurgia ortognática: } \\
\text { ensaio clínico randomizado' (SOUSA, 2016) }\end{array}$ & $\begin{array}{l}\text { Universidade de } \\
\quad \text { São Paulo }\end{array}$ \\
\hline \multirow{3}{*}{$\begin{array}{l}\text { "Educação em saúde" } \\
\text { AND enfermagem } \\
\text { AND "Pós-operatório" } \\
\text { AND Pacientes AND } \\
\text { "Educação de pacientes } \\
\text { como assunto" }\end{array}$} & $\mathrm{D}$ & 2013 & $\begin{array}{l}\text { Tecnologia educativa de cuidados para o pós- } \\
\text { operatório da cirurgia de revascularização } \\
\text { miocárdica: uma ferramenta para o paciente e } \\
\text { família (GENTIL, 2013) }\end{array}$ & $\begin{array}{l}\text { Universidade de } \\
\quad \text { São Paulo }\end{array}$ \\
\hline & $\mathrm{D}$ & 2017 & $\begin{array}{l}\text { Orientações na alta hospitalar a partir das } \\
\text { necessidades sentidas por pacientes submetidos à } \\
\text { cirurgia ortopédica (ITO, 2017) }\end{array}$ & $\begin{array}{l}\text { Pontifícia } \\
\text { Universidade } \\
\text { Católica de São } \\
\text { Paulo }\end{array}$ \\
\hline & $\mathrm{D}$ & 2013 & $\begin{array}{c}\text { O processo de educação em saúde em um grupo de } \\
\text { orientação de alta às mulheres mastectomizadas } \\
\text { (BORDALLO, 2013) }\end{array}$ & $\begin{array}{c}\text { Universidade } \\
\text { Federal Fluminense }\end{array}$ \\
\hline
\end{tabular}

Legenda: tese (T), dissertação (D). Fonte: Bittencourt; Schwengber; Stumm, 2021. Fonte: Autores.

O maior número de publicações ocorreu em 2013, com quatro trabalhos (44,4\%). Houve predomínio de estudos nos programas de pós-graduação stricto sensu da região sudeste do país, com oito pesquisas (88,9\%), seguida da região nordeste $(11,1 \%)$. Cabe mencionar que nas regiões sul, norte e centro-oeste não foram identificadas teses e dissertações sobre a temática investigada, o que reforça lacuna existente de estudos na região sul do Brasil, local em que a universidade das autoras desse texto está inserida.

Quanto à área de concentração dos programas de pós-graduação stricto sensu, foram contabilizados oito (88,9\%) estudos desenvolvidos em programas da grande área Ciências da Saúde, seguidos por cinco na área da Enfermagem, dois na 
Enfermagem da Saúde do Adulto e um na Enfermagem Fundamental. Outro estudo $(11,1 \%)$ pertence a área Multidisciplinar, Mestrado Profissional em Educação nas Profissões da Saúde.

Com relação às instituições de ensino às quais os pesquisadores estão vinculados, verificou-se maior número de pesquisas produzidas pelo Programa de Pós-Graduação em Enfermagem da Universidade de São Paulo, com três (33,3\%) trabalhos, seguido pelo Programa de Pós-Graduação em Enfermagem da Universidade Federal Fluminense, com dois (22,2\%).

No que se refere à abordagem metodológica dos estudos, três (33,3\%) são quantitativos, três (33,3\%) qualitativos e três $(33,3 \%)$ mistos. Os tipos de pesquisa mais utilizadas foram ensaio clínico controlado (33,3\%) e estudo descritivo (33,3\%). As ações educativas foram direcionadas para pacientes com indicações cirúrgicas variadas, como cardíaca (22,2\%), ortopédica $(22,2 \%)$, bariátrica $(11,1 \%)$, transplante renal $(11,1 \%)$, prostatectomia radical $(11,1 \%)$, ortognática $(11,1 \%)$ e mastectomia $(11,1 \%)$. Com relação ao período cirúrgico em que a ação educativa foi desenvolvida, os maiores percentuais foram de pesquisas no pós-operatório $(55,6 \%)$, seguida por duas $(22,2 \%)$ no pré-operatório e duas $(22,2 \%)$ no pré e pós-operatório.

Em todas as pesquisas foram criados produtos para o desenvolvimento das ações educativas ou as autoras construíram e validaram materiais educativos, também denominados como produtos tecnológicos em algumas das pesquisas (SOUSA, 2016; Borsato, 2014; Gentil, 2013). Os produtos tecnológicos educativos utilizados ou criados, resultantes da revisão narrativa, foram quatro cartilhas $(44,4 \%)$, dois livretos com orientação oral $(22,2 \%)$, um vídeo $(11,1 \%)$, um infográfico $(11,1 \%)$ e um dos estudos explicitou o uso de tecnologias expositivas/dialógicas e impressas $(11,1 \%)$.

Todos os estudos analisados apresentam palavras-chave e/ou descritores com, pelo menos, um descritor exato ou sinônimo, conforme os Descritores em Ciências da Saúde (DeCS). Foram contabilizadas 24 palavras-chave e/ou descritores na soma dos estudos, com destaque para os descritores "enfermagem" e "educação em saúde", ambos mencionados em cinco (55,5\%) trabalhos. Outros descritores mais utilizados foram: "alta do paciente", "tecnologia educacional" e "enfermagem perioperatória", estes últimos três descritores estavam presentes em conjunto em três (33,3\%). Das 24 palavras-chaves e/ou descritores identificadas, 17 (70,83\%) eram descritores cadastrados nos DeCS e sete $(29,17 \%)$ palavras-chave.

Barros (2017) avaliou a efetividade de intervenção educativa mediada pela cartilha "Cirurgia bariátrica: cuidados para uma vida saudável” no preparo pré-operatório. Trata-se de um ensaio clínico randomizado controlado, em que os grupos controle e intervenção foram seguidos por sete semanas. O grupo de intervenção participou de uma ação educativa mediada pela cartilha e a percepção dos pacientes foi de melhora na ansiedade e depressão. Tais achados são reafirmados por Han et al. (2018), que em uma população diferente, pacientes no pós-operatório de câncer laríngeo, constataram melhora significativa na qualidade de vida e redução de experiências negativas após ações educativas no grupo de intervenção.

Os participantes da pesquisa de Barros (2017) referiram, ainda, satisfação com o conhecimento adquirido durante a intervenção educativa, como informações suficientes sobre cirurgia, anestesia, pós-operatório hospitalar e domiciliar da cirurgia bariátrica. A qualidade das informações demonstra a possibilidade de que o instrumento educativo agrega com a elucidação de cuidados e promove segurança aos pacientes. O tempo de acompanhamento dos pacientes com a utilização de ação educativa também pode ser um diferencial nos resultados obtidos se comparados a pacientes que recebem orientações verbais rotineiras (Barros et al., 2020).

Nesse contexto, durante o perioperatório é possível identificar o nível de entendimento sobre a cirurgia, sentimentos e expectativas relacionados ao tratamento cirúrgico e do impacto da cirurgia em relação ao estado de saúde geral do paciente, além de promover um espaço dialógico. Tal afirmativa vai ao encontro de Pinto (2016), que descreveu, analisou o uso das tecnologias educacionais e compreendeu o significado destas na assistência perioperatória no contexto da consulta de enfermagem na perspectiva de enfermeiros, pacientes e familiares. Os pacientes relataram que aprenderam os cuidados de saúde na prática educativa, nos encontros com os enfermeiros, e com isso sentiram-se satisfeitos, seguros e confiantes para 
participarem do tratamento cirúrgico. Outra pesquisa também constatou que um programa educativo foi avaliado pelos participantes como importante para garantir adesão aos cuidados, tranquilidade e auxílio na recuperação (Rosseto et al., 2017).

A atenção dos enfermeiros para com o interesse e aceitação do paciente em participar da educação em saúde também foi levantada por Rosseto (2013), a qual reforça a percepção e o desenvolvimento da melhor abordagem. A referida dissertação teve como objetivo analisar, sob a ótica de pacientes submetidos à cirurgia cardíaca, as contribuições de um programa educativo de enfermagem no pré-operatório. A autora constatou que os participantes referiram a necessidade de uma melhor abordagem quanto às possíveis intercorrências, sobre o eventual direcionamento do paciente para uma Unidade de Terapia Intensiva no pós-operatório imediato, uso de sonda vesical de demora e ações educativas diferenciadas para pacientes com menor nível de escolaridade.

A relevância da observação do aceite e interesse do paciente em participar ativamente da ação educativa é ressaltada por Rosseto (2013) e vem ao encontro de uma pesquisa desenvolvida por enfermeiros em um departamento de enfermagem na China. Nesta constatou-se a necessidade de alinhar as informações trabalhadas na educação em saúde, dos pacientes submetidos à cirurgia de intervenção coronariana percutânea de forma individualizada, com incentivo e apoio no interesse dos pacientes em participar de todo o processo, a começar pelo pré-operatório (Zhuo et al., 2020).

A coerência do desenvolvimento de ações educativas foi revelada também por Borsato (2014) que, dentre os objetivos, avaliou a percepção do paciente transplantado renal sobre as orientações de enfermagem no momento da alta hospitalar e constatou que informações sobre como se cuidar em casa e preservar o enxerto eram fornecidas pela enfermagem de maneira assistemática. A autora destacou a falta de tempo e de pessoal para a realização de orientações de forma adequada, ausência de padronização para as orientações de enfermagem ao paciente em todo o processo de transplante renal e a falta de comunicação entre a equipe de enfermagem e a equipe médica.

Ainda, Mata (2013) e Borsato (2014) não relacionaram diretamente suas pesquisas com a segurança dos pacientes. Todavia, evidenciaram, sequencialmente, melhorias no conhecimento quanto aos cuidados e satisfação dos pacientes (Mata, 2013), e enfatizaram que os pacientes são admitidos para procedimentos cirúrgicos sem possuírem conhecimento contundente acerca dos mesmos, tampouco os enfermeiros os esclarecem de forma satisfatória (Borsato, 2014).

O estudo experimental de Mata (2013) avaliou a efetividade de um programa educativo para o cuidado domiciliar de pacientes submetidos à prostatectomia radical, apoiado nas seguintes dimensões: autoeficácia geral e percebida, ansiedade, ansiedade somada à depressão, satisfação e conhecimento de pacientes. A referida intervenção resultou em mudança clínica relevante, no que se refere ao conhecimento adquirido pelos pacientes, por meio do programa educativo. A autora concluiu que o fato de a intervenção utilizar combinação de orientação oral, escrita e acompanhamento telefônico mostrou-se efetiva no âmbito dos cuidados em domicílio e da satisfação dos indivíduos. O referido estudo foi incluído nessa revisão pelo fato de o primeiro contato com os participantes ter ocorrido ainda na internação hospitalar, o que fortalece a coerência de investir em ações educativas em saúde na hospitalização.

A diminuição tanto da ansiedade, depressão quanto dos níveis da dor foram percebidos em uma revisão integrativa desenvolvida com o objetivo de analisar a literatura científica produzida sobre métodos de avaliação da efetividade de tecnologias educativas para paciente cirúrgicos (Paiva; Sousa; Poveda; Turrini, 2017).

A educação do paciente por meio de material educativo contribuiu para uma intervenção eficiente, é necessário que o material seja de fácil acesso, com linguagem adequada ao público-alvo, e que permita consulta durante as etapas do perioperatório. Informar a respeito da técnica e sobre o procedimento ao qual a pessoa será submetida contribui de forma significativa para uma melhor recuperação.

Bordallo (2013) também contatou sua população, mulheres mastectomizadas, no último dia da internação hospitalar e continuou o acompanhamento no pós-operatório de um grupo educativo. O objetivo da pesquisa foi conhecer o processo de 
educação em saúde realizado no grupo de orientação de alta na perspectiva das mulheres mastectomizadas, identificar conhecimentos apreendidos por elas após o grupo de orientação para alta e discutir os conteúdos da educação em saúde a partir de suas demandas. Os resultados da pesquisa possibilitaram sanar dúvidas relacionadas aos cuidados pós-operatórios, à volta às suas atividades de vida diária e à continuidade do tratamento por meio do desenvolvimento de um vídeo educativo durante o grupo. Este facilitou a construção do conhecimento e segurança por se tratar de um material educativo com recursos visuais e auditivos (Bordallo, 2013). O autor concluiu, ainda, que os saberes podem ser instigados aos pacientes para uma real construção e reconstrução conjunta com o educador, situação em que ambos se encontram inseridos no processo de cuidar e educar em saúde.

Um ensaio clínico randomizado controlado, para avaliar a eficácia de uma tecnologia educativa impressa na redução de sinais e sintomas perioperatórios em pacientes submetidos à cirurgia ortognática mostrou que a ação educativa foi efetiva para o conhecimento de pacientes sobre a cirurgia, autocuidado, segurança, melhora dos desfechos avaliados e considerou que a tecnologia educativa auxilia no pós-operatório (Sousa, 2016).

A participação de pacientes e familiares foi considerada por Gentil (2013), na elaboração de uma ferramenta educativa para auxílio no autocuidado de paciente revascularizado e após a alta hospitalar. Os participantes do referido estudo auxiliaram na construção da ferramenta ao apontar a necessidade de abordagens sobre o uso correto dos medicamentos, cuidados com alimentação, com a ferida operatória, identificação de intercorrências e direcionamentos a serviços de saúde, retorno à consulta médica e enfrentamento das dificuldades no pós-operatório.

Uma ação educativa também foi desenvolvida para a alta hospitalar por Ito (2017), que objetivou conhecer os sentimentos de pacientes submetidos à cirurgia ortopédica ao receber alta hospitalar, identificar as necessidades de orientação sentidas por esses pacientes, conhecer suas percepções durante a alta hospitalar, identificar as profissionais envolvidas e construir coletivamente um material didático para paciente e família. Foi constatado que o planejamento da alta se dá com base nas necessidades do paciente, inclui seu aprendizado, abrange desde a admissão até a alta hospitalar, com vistas a tornar essa mais segura para o paciente. Porém, $45 \%$ dos participantes referiram que os enfermeiros não participaram do planejamento da alta de forma sistemática (ITO, 2017).

A revisão narrativa possibilitou conhecer diferentes metodologias que podem ser utilizadas para a realização de ações educativas por enfermeiros ao paciente hospitalizado no perioperatório. Ela destacou também a necessidade da presença dos enfermeiros na ação educativa para a segurança dos pacientes.

\section{Conclusão}

A pesquisa possibilitou conhecer estudos que tematizam ações educativas acerca da segurança dos pacientes no perioperatório desenvolvidas na hospitalização, porém as produções selecionadas não deixam claro se os pacientes relacionam as ações educativas em saúde com a segurança. A análise dos estudos que integraram essa revisão demonstra a lacuna de investigações que contemplem ações educativas por enfermeiros direcionadas à segurança do paciente cirúrgico.

Um aspecto merecedor de reflexões e ações dos enfermeiros que atuam no perioperatório reside no fato de o paciente referir que os enfermeiros necessitam estar mais presentes nas ações educativas. Nesse sentido, essa pesquisa possibilita incentivar os enfermeiros a pensar sobre o planejamento e implementação de ações educativas no perioperatório, com vistas a ampliar o conhecimento do paciente, dirimir dúvidas, promover conforto, bem-estar e segurança. Para que isso ocorra, requerse interação, dialogicidade e vínculo entre paciente cirúrgico e enfermeiro, a qual resultará em construção do conhecimento de ambos os atores envolvidos. Entende-se que o paciente sente a necessidade de aporte de conhecimento pela situação que vivencia, logo, se constitui em um momento adequado de ações dos enfermeiros para contribuir na recuperação dele. 


\section{Referências}

Barros, L. M. (2017). Efetividade da cartilha "cirurgia bariátrica: cuidados para uma vida saudável” no preparo pré-operatório: ensaio clínico randomizado. 2017. 240 p. Tese (Doutorado em Enfermagem) - Universidade Federal do Ceará, Fortaleza.

Barros, L. M. et al . (2020). Intervenção educativa e indicadores de obesidade de candidatos à gastroplastia: estudo quase-experimental. Acta paul. enferm., São Paulo, v.33, eAPE20180305.

Böck, A. et al. (2019). Ações educativas desenvolvidas no período perioperatório em um hospital universitário: percepção de pacientes cirúrgicos. Rev. enferm. UFSM., v. 9, n. 28, p. 1-20.

Bordallo, F. R. (2013). O processo de educação em saúde em um grupo de orientação de alta às mulheres mastectomizadas. 121 p. Dissertação (Mestrado Profissional em Enfermagem) - Universidade Federal Fluminense, Niterói.

Borsato, L. (2014). Cartilha com orientações de enfermagem para a alta hospitalar: contribuição à educação em saúde do paciente transplantado renal. 2014. 134 p. Mestrado (Mestrado Profissional em Enfermagem) - Universidade Federal Fluminense, Niterói.

Brasil. (2014). Ministério da Saúde, Fundação Oswaldo Cruz, Agência Nacional de Vigilância Sanitária. Documento de referência para o Programa Nacional de Segurança do Paciente. Brasília: Ministério da Saúde.

Brum, C. N. et al. (2016). Revisão narrativa de literatura: aspectos conceituais e metodológicos na construção do conhecimento da enfermagem. In: LACERDA, M. R.; COSTENARO, R. G. S. (org.). Metodologias da pesquisa para a enfermagem e saúde: da teoria à prática. Porto Alegre: Mória.

Gentil, L. L. S. (2013). Tecnologia educativa de cuidados para o pós-operatório da cirurgia de revascularização miocárdica: uma ferramenta para o paciente e família. 161 p. Dissertação (Mestrado em Enfermagem no cuidado do adulto) - Universidade de São Paulo, São Paulo.

Han, J. et al. (2018). Effects of health education intervention on negative emotion and quality of life of patients with laryngeal cancer after postoperative radiotherapy. Cancer radiother, v. 22, n. 1, p. 1-8.

Ito, N. A. P. (2017). Orientações na alta hospitalar a partir das necessidades sentidas por pacientes submetidos à cirurgia ortopédica. 89 p. Dissertação (Mestrado Profissional em Educação nas Profissões da Saúde Instituição de Ensino) - Pontifícia Universidade Católica de São Paulo, Sorocaba.

Mata, L. R. F. D. (2013). Efetividade de um programa de ensino para o cuidado domiciliar de pacientes submetidos à prostatectomia radical: ensaio clínico randomizado. 2013. 191 p. Tese (Doutorado em Enfermagem) - Universidade de São Paulo, São Paulo.

Paiva, B. C., Sousa, C. S., Poveda, V. B., \& Turrini, R. N. T. (2017). Avaliação da efetividade da intervenção com material educativo em pacientes cirúrgicos: revisão integrativa da literatura. Rev. Sobecc, São Paulo. v.22, n.4, p.208-217.

Pinto, A. C. S. (2016). Tecnologias educacionais na assistência perioperatória em cirurgia ortopédica no contexto da consulta de enfermagem. 193 p. Tese (Doutorado em Enfermagem) - Universidade Federal do Rio de Janeiro, Rio de Janeiro.

Rosseto, C. K. R. (2013). Influência do processo educativo de enfermagem no pré e pós-operatório de paciente de cirurgia cardíaca. 101 p. Dissertação (Mestrado Profissional em Enfermagem Instituição de Ensino) - Centro de Ciências da Saúde, Universidade Federal do Espírito Santo, Vitória.

Rosseto, C. K. R. et al. (2017). Intervenção educativa de enfermagem ao cliente submetido à cirurgia cardíaca. Rev. baiana enferm., Salvador, v.31, n. 4, e22441.

Sousa, C. S. (2016). Avaliação do uso de uma tecnologia educativa na educação perioperatória de cirurgia ortognática: ensaio clínico randomizado. 135 p. Tese (Doutorado em Enfermagem) - Universidade de São Paulo, São Paulo.

Vasconcelos, E. M. (2019). Educação popular em saúde: constituição e transformação de um campo de estudos e práticas na saúde coletiva. In: PELICIONI, M. C. F.; MIalhe, F. L. Educação e promoção da saúde: teoria e prática. 2. ed. Rio de Janeiro: Santos.

Vasconcelos, E. M.; Cruz, P. J. S. C.; \& Prado, E. V. (2016). A contribuição da Educação Popular para a formação profissional em saúde. Interface comun. saúde educ., v. 20, n. 59, p. 835-838.

Who. (2008). World Health Organization. World Alliance for Patient Safety. Forward Programme 2008-2009. Geneva: WHO.

Zhuo, Q. et al. (2020). Perceptions of patients undergoing percutaneous coronary intervention on pre-operative education in China: a qualitative study. Health Expectations, v. 00, n. 1, p. 1-10. 